\title{
Neoclassical simulations of fusion alpha particles in pellet charge exchange experiments on the Tokamak Fusion Test Reactor
}

\author{
M. H. Redi ${ }^{\text {a }, ~ S . ~ H . ~ B a t h a, ~ M . ~ G . ~ B e l l, ~ R . ~ V . ~ B u d n y, ~ D . ~ S . ~ D a r r o w, ~}$ \\ F. M. Levinton, D. C. McCune, \\ S. S. Medley, M. P. Petrov ${ }^{b}$, S. von Goeler, R. B. White, M. C. Zarnstorff, \\ S. J. Zweben and the TFTR Team \\ Princeton Plasma Physics Laboratory, Princeton University, Princeton, NJ, 08543
}

\begin{abstract}
Neoclassical simulations of alpha particle density profiles in high fusion power plasmas on the Tokamak Fusion Test Reactor (TFTR) [Phys. Plas. 5, 1577 (1998)] are found to be in good agreement with measurements of the alpha distribution function made with a sensitive active neutral particle diagnostic. The calculations are carried out in Hamiltonian magnetic coordinates with a fast, particle-following Monte Carlo code which includes the neoclassical transport processes, a recent first-principles model for stochastic ripple loss and collisional effects. New global loss and confinement domain calculations allow an estimate of the actual alpha particle densities measured with the pellet charge exchange diagnostic.
\end{abstract}

PACS: 52.25.Fi, 52.55.Fa, 52.55.Pi, 52.65.-y

\footnotetext{
${ }^{\mathrm{a}}$ redi@princeton.edu

${ }^{\mathrm{b}}$ Permanent address: Ioffe Physical-Technical Institute, St. Petersburg, Russia 194021.
} 


\section{INTRODUCTION}

Confinement of the energetic charged fusion products of thermonuclear reactions will be essential to sustain ignition in a magnetic fusion reactor. Confinement of the thermal ions is also essential for maintaining a steady state, high performance plasma. Thermal ion transport has been found to be much higher than expected from neoclassical theory and is not yet well understood. In contrast, it has been argued that energetic ion transport will be well described by neoclassical plasma physics because energetic ions move too fast and have too large a gyroradius to be influenced by microinstabilities and short wavelength turbulence in the thermal plasma. Transport analysis of experiments at the Tokamak Fusion Test Reactor (TFTR) [1] based on neoclassical confinement of suprathermal ions reproduces measured plasma stored energy and neutron emission. Furthermore, guiding center codes are able to match the global loss rates of fusion products inferred from triton burnup experiments at JT-60U [2]. Both these observations support the assertion that suprathermal ions behave classically in the single particle limit. Of particular interest for fusion reactor planning have been the so-called "reversed shear" (see Sec. II) equilibria in tokamaks. These plasmas exhibit unusually high thermal and particle confinement and are considered promising potential reactor candidates.

In this paper we extend neoclassical computer simulations of global alpha particle transport [3] in monotonic and reversed shear equilibria, to predict radially resolved density profiles for confined alpha particles in TFTR. These simulations are compared to profiles directly measured with the pellet charge exchange diagnostic (PCX) $[4,5]$. 
The agreement of these simulation results with the measurements of confined alpha profiles as well as the agreeement of Lorentz code modelling with alpha loss data [6] directly validates neoclassical modeling of energetic ions in toroidal configurations. Accurate calculation of confined fast ion distributions with computational tools benchmarked against measurements will allow minimized reactor and magnetic coil system size leading to a more economical reactor design. The paper is divided into four sections. In Section II the simulation procedure and experimental scenarios are discussed. Results are presented in Section III with a conclusion and summary in Section IV.

\section{SECTION II. SIMULATION METHOD AND EXPERIMENTAL SCENARIOS}

Guiding center code simulations have been carried out to model energetic ion transport of experiments on TFTR in high power, good confinement plasmas with similar major and minor radii, toroidal field, final levels of neutral beam power, plasma current and edge q. In Ref. 3 we carried out global loss simulations of alpha loss for deuterium-tritium (DT) projections of deuterium-deuterium experiments in monotonic and reversed shear configurations. PCX data was subsequently obtained in DT plasmas that were similar but not identical to the plasmas previously studied computationally. In Table I are shown the parameters describing both these scenarios. The simulations of the PCX experimental radial profiles described in this paper use the earlier plasma parameters, which were obtained from plasma data analysis with the TRANSP analysis code [7] and equilibrium calculations made with the PEST code [8] close to the end of the neutral beam heating. In this section and in Sec. IIIA we compare the confinement 
domains and global losses calculated for these two sets of reversed and monotonic shear scenarios. These comparisons, given TRANSP simulation of the PCX DT experiment alpha fusion power, allow us to estimate the alpha particle densities measured with PCX.

Each point in a toroidal plasma magnetic equilibrium can be designated by a parameter $\mathrm{q}=\mathrm{d} \Phi / \mathrm{d} \Psi$, the rate of change of the toroidal flux $\Phi$ with poloidal flux $\Psi$; the magnetic shear, $\mathrm{s}=(\mathrm{r} / \mathrm{q}) \mathrm{dq} / \mathrm{dr}$, is the dimensionless radial derivative of this plasma safety factor, q. The q profiles used in the simulations of Ref. 3 and the PCX experiments are shown in Fig. 1, labeled monotonic shear ( $\mathrm{s}>0$ everywhere) and reversed shear ( $<<0$ in the core). The major radii of the magnetic axes in the PCX experimental plasmas are smaller than for the plasmas simulated in Ref. 3, which were based on DT projections of TFTR DD experiments. MSE measurements of $q(\mathrm{r})$ were not available for the monotonic shear plasma in which PCX measurements were made, but TRANSP q(r) calculations based on neoclassical resistivity are generally in good agreement with MSE for similar experiments near the end of the beam phase. The q profile calculated by TRANSP during the early part $(2.2 \mathrm{sec})$ of the high power beam heating phase of the reversed shear experiment in which the PCX measurements were made are shown in Figure 2. This profile is similar to the q profile measured with motional Stark effect (MSE) for the reversed shear experiment outside $\mathrm{r} / \mathrm{a} \sim 0.2$. The subsequent evolution of the q profile is expected to also be similar in TRANSP simulation and in the experiment

Reversed shear plasmas, with high central q and non-monotonic q profiles can exhibit remarkably reduced energy and particle transport of the thermal ions. However, these same conditions are theoretically predicted to produce high levels of stochastic 
ripple loss of suprathermal particles (the threshold criterion for ripple loss, defined below, $\sim \mathrm{q}^{-5 / 2}$ ). This will potentially reduce the efficiency of plasma heating by alpha particles and of other heating schemes involving fast ions. Note that these are large major radius plasmas for which toroidal magnetic field ripple is most significant in TFTR.

For the pellet charge exchange measurement, low $\mathrm{Z}$ impurity pellets, lithium or boron, are injected along a midplane major radius. A small fraction of the alphas incident on the neutral cloud surrounding the ablating pellet is neutralized by sequential single electron capture or by double electron capture. By measuring the energy distribution of the resultant helium neutrals escaping from the plasma as a function of time, the energy distribution of the incident alpha particles can be determined as a function of radius.

\section{A. Simulations with the ORBIT guiding center code}

Simulations were carried out with ORBIT [9], a Hamiltonian coordinate, Monte Carlo guiding center code. ORBIT follows an ensemble of alpha particles, initially at 3.5 MeV, in a realistic toroidal magnetic geometry for one alpha particle slowing down time. The simulations incorporate the collisional effects of pitch angle scattering (rate denoted by $v_{\mathrm{pa}}$ ) and energy loss due to collisional drag (energy slowing down collision rate denoted by $v_{\varepsilon}$ ) as well as toroidal field ripple and first orbit loss. The calculations are speeded up by 1) a two-dimensional spline fit to the tokamak geometry, 2) a newly developed, rapid, first principles algorithm [10] for the spatial location of the stochastic confinement domain within the plasma and 3) a technique for collisional acceleration based on the time scale separation between collisions and the orbit bounce motion. Both the monotonic shear plasmas, which exhibited enhanced confinement (supershot 
performance), and reversed shear plasmas had peaked alpha particle density profiles. The birth alpha profiles were modeled as $\left(1-(\mathrm{r} / \mathrm{a})^{2}\right)^{9}$ and $\left.(1-\mathrm{r} / \mathrm{a})^{2}\right)^{5}$ for monotonic and reversed shear respectively. These initial alpha particle radial profiles, deduced from fits to TRANSP predicted alpha density profiles, agree with the alpha birth profile in similar deuterium-tritium experiments as measured by the neutron collimator on TFTR.

\section{B. Stochastic criterion and confinement domains}

White has developed a new, first principles, analytic model for the toroidal field ripple diffusion stochastic loss criterion [11] as well as an equivalent rapid, accurate computational algorithm [10]. These new methods are much improved over the usual zero banana width formulation of Goldston, White, and Boozer (GWB) [12]. Trapped ion loss from a tokamak occurs due to toroidal field line ripple when the orbiting ion reaches its banana tip and the threshold criterion $\delta_{\mathrm{TP}} / \delta_{\mathrm{s}}>1$ is satisfied, producing stochastic diffusion. Here $\delta_{\mathrm{TF}}$ is a measure of the average toroidal field line ripple

$$
\delta_{\mathrm{TF}}=\left(\mathrm{B}_{\mathrm{MAX}}-\mathrm{B}_{\mathrm{MIN}}\right) /\left(\mathrm{B}_{\mathrm{MAX}}+\mathrm{B}_{\mathrm{MIN}}\right)
$$

and $\delta_{\mathrm{s}}$ is a stochastic threshold similar to the well known GWB stochastic threshold

$$
\delta_{\mathrm{GWB}}=(\varepsilon / \mathrm{N} \pi \mathrm{q})^{3 / 2}\left(1 / \rho \mathrm{q}^{\prime}\right) .
$$

$\mathrm{N}$ is the number of toroidal field coils, $\rho$ is the ion gyro radius, $\varepsilon$ is the inverse aspect ratio $\mathrm{a} / \mathrm{R}$ and $\mathrm{q}^{\prime}=\mathrm{dq} / \mathrm{dr}$. The simple model threshold $\delta_{\mathrm{GWB}}$ does not include dependence on poloidal angle or variation of toroidal precession of the energetic ions. The first principles algorithm and analytic model used here includes these effects, which have been shown to modify the $\delta_{\mathrm{GWB}}$ stochastic threshold by a factor of 10 when comparing alpha

particles to neutral beam loss in the same plasma $\left(\delta^{\alpha}{ }_{\mathrm{s}} \sim 0.6 \delta^{\alpha}{ }_{\mathrm{GWB}}, \delta^{\mathrm{nb}}{ }_{\mathrm{s}} \sim 4 \delta^{\mathrm{nb}}{ }_{\mathrm{GWB}}\right)$ 
[13]. An ion satisfying the criterion is deleted from the calculation, reducing the number of calculations required to only those ions which will remain confined. In 1995, a prediction of the alpha distribution function for a high power TFTR DT monotonic shear experiment was obtained by Budny et al. [14] using neoclassical simulations within the TRANSP analysis code including a simple model for ripple loss [13]. We subsequently compared PCX measurements with the TRANSP alpha profile to show the effect of a GWB ripple model in a monotonic shear plasma [13].

\section{SECTION III. RESULTS AND DISCUSSION}

\section{A. Simulation Results for PCX Experimental Parameters}

Figure 3 shows the calculated confinement domains for alpha particles at birth energy in the monotonic and reversed shear geometries of the PCX experiments with the new confinement domain formulation. In reversed shear, most of the plasma is above threshold for stochastic ripple loss of trapped alpha particles at the birth energy, 3.5MeV. In the simulations of the PCX reversed shear experiment, $33 \%$ of the ions are lost if there is no pitch angle scattering. Of this $25 \%$ is prompt loss and $8 \%$ is collisionless stochastic ripple loss which takes place slowly over the slowing down time.

This is in contrast to the reversed shear scenario simulated in Ref. 3, with higher $q(0)$, for which all of the initially trapped alphas were lost rapidly through unconfined orbit losses or collisionless stochastic ripple. The effect of pitch angle scattering of passing particles refills the trapped distribution and leads to continued alpha loss throughout the slowing process in both reversed shear scenarios. 
Figure 4 shows the evolution of the alpha particle loss predicted for the PCX experiments over one slowing down time. The predicted losses for reversed shear ( $40 \%$ ) are smilar to those in Ref. 3, but and $\sim 6$ times the total alpha losses predicted for the monotonic shear case. In monotonic shear cases, the processes of stochastic ripple diffusion and pitch angle scattering were found to combine nonlinearly; pitch angle scattering due to collisions enhanced ripple loss by a factor of 2 [15]. This strong synergism between collisions and ripple transport, calculated for high current, monotonic shear plasmas, is not, however, found in reversed shear because of high first orbit loss and strong collisionless ripple diffusion [3].

Global loss simulation results for the magnetic geometry and the collision rates of the PCX experiments are shown in Table II in addition to those calculated for the scenarios of Ref. 3. It is found that first orbit losses (from an ensemble of 256 particles) for the monotonic and reversed shear plasmas were $4 \%$ and $20 \%$ and that over one energy slowing down time the accumulated particle losses were $7 \%$ and $39 \%$ respectively. The monotonic shear global losses are less than in the earlier simulations, primarily because of smaller values for the major and minor radii, $\mathrm{q}$ and $v_{\mathrm{pa}}$ in the actual experiment. The strong effects of a, $\mathrm{R}_{\mathrm{axis}}$ and $\mathrm{q}$ on TF ripple diffusion are shown by the $3 \%$ delayed alpha loss in the simulation of the PCX monotonic shear experiment versus the 17\% loss in the simulation of the earlier monotonic shear scenario. The reversed shear global loss fractions are nearly unchanged from the earlier set of simulations because $\mathrm{q}$ is so high in both reversed shear scenarios.

Most different between the sets of parameters in Table I are the collision rates for pitch angle scattering and slowing down. The pitch angle scattering rate in the Ref. 3 
monotonic shear simulation is four times that of the PCX experiment. As a result, 93\% of the alphas are retained in simulation of the monotonic shear PCX experiment, while only $77 \%$ are retained in the monotonic shear simulation of Ref. 3 . This affects the magnitude of the simulated confined alpha density for the PCX experiment, leading to an underestimate of the confined density by $\sim 20 \%$ after one slowing down time. It would not modify the radial profile prediction. The simulations assumed radially constant collision rates. Including a radial variation in the collision rate is expected to make only small differences in the results because the ratio of the pitch angle scattering rate to the energy slowing down rate is nearly constant with radius, according to TRANSP analysis. This approximation has been shown not to change global loss predictions [3].

ORBIT simulations have shown [3] that the effects of collisional ripple loss are distributed evenly across the alpha profile, not limited to edge or central regions of high q, so that we expect the predicted alpha profiles to correspond to the experimental profiles. A separate set of sensitivity study simulations to test collision models was run for the reversed shear case with $v_{\varepsilon}$ reduced to $4 / \mathrm{sec}$. No change in the alpha profiles was found.

\section{B. Radial profile simulations}

This paper focusses on radially resolved alpha density profiles whose calculation require several orders of magnitude more computation than the global loss calculations of the previous Section. The radial profiles were simulated with the

parameters of the scenarios studied in Ref. 3, as an extension of those calculations. The 
effect on the predicted profiles of differences between the simulation parameters to those of the experiments is discussed in Sec. IIIC.

Because the PCX diagnostic has an observation window which detects only very deeply trapped alphas with pitch $\lambda=\mathrm{v}_{/} / \mathrm{v}=-0.05 \pm 0.001$ at the midplane, it was necessary to follow a very large initial ensemble of alpha particles to obtain adequate statistics within a window of constrained pitch to model the diagnostic. For monotonic shear plasmas an initial ensemble of 50,000 alphas was sufficient (as compared to 256 alphas for global estimates of alpha loss rates), taking $\sim 4$ hours Cray C90 cpu time. In the reversed shear plasma being simulated on TFTR, however, all of the trapped alphas are lost at birth. Only alphas born on passing orbits and later pitch-angle scattered into trapped orbits can be seen by PCX as they slow down, so that it was necessary to simulate $\sim 2,000,000$ alpha particles to obtain a statistically significant count of 400 alphas within the simulated data collection window, with $\lambda=-0.15$ to +.05 at the midplane.

To minimize computational time, ORBIT used the analytic stochastic loss algorithm and accelerated the collision rates, $v_{\mathrm{pa}}$ and $v_{\varepsilon}$, by a factor of 100 with a reduced run time, $\tau_{\varepsilon}=1 / \nu_{\varepsilon}, \tau_{\varepsilon} \rightarrow \tau_{\varepsilon} / 100$. This method of speeding up the loss calculations has been shown to give good global loss estimates even for reversed shear equilibria [3]. Simulations of monotonic shear using the TRANSP code with accelerated collisions [13] indicate that the technique can be used for alpha particles but not for accurate neutral beam ion calculations. Use of conservation laws for ion energy and magnetic moment was also used to project the final orbits to $\theta=0$ to further improve statistics. This required $\sim 300$ hours of cpu time. Without the rapid calculation features of the new code, these 
calculations would not have been possible; 30,000 hours ( 8 years) of cpu time would have been needed to simulate the reversed shear alpha profiles. Carrying out the radial profile simulations with the parameters of the configurations of the actual PCX experiments would take an additional six months of calculation and is not feasible.

\section{Comparison with PCX data}

Figures 5 and 6 present a comparison of the simulated radial alpha density profiles to PCX analyzed data for the monotonic and reversed shear scenarios for two different alpha particle energies. The ORBIT simulation energies were chosen to be the alpha energy after one and two energy slowing down times: $1.3 \mathrm{MeV}$ and $0.5 \mathrm{MeV}$, energies corresponding to characteristic transport times of the experiment. The simulation results were previously compared to the same PCX data analyzed at alpha energies of 0.5-0.6 MeV, 1.0 MeV and 1.7 MeV [16]. This earlier analysis of PCX data did not show good agreement with the guiding center code results at the higher alpha energies in reversed shear. In particular, a reduced alpha density or "hole in the distribution" was found at $1.7 \mathrm{MeV}$. More recent papers $[17,18]$ compare the simulation results with PCX data analyzed at energies close to the ORBIT simulation alpha particle energies. All of these earlier references [16-18] relied on the stochastic confinement domain and global loss predictions from Ref. 3, which differ from calculations for the actual experiment as detailed in Sec. IIIA.

The comparison of theoretical prediction to data in the figures has been further improved since these earlier papers, as the vertical scale for the comparison is no longer given in arbitrary units, but on a scale of alpha particle density within restricted ranges of 
pitch angle and energy at the midplane. The PCX data in each scenario is separately normalized to a fit to the ORBIT simulated profiles at the radius shown. The ORBIT alpha densities are obtained from TRANSP calculations of the fusion alpha birth rate, integrated over times corresponding to the energy resolution of the PCX diagnostic (5$11 \%)$ and scaled by the average fraction of alphas retained in the monotonic $(734 / 50,000)$ and reversed shear $(62 / 50,000)$ simulations. The densities are also reduced to correspond to the PCX pitch acceptance window, as the PCX measurement window is $1 / 100$ th the size of the ORBIT pitch acceptance window. The simulated confined alpha density in monotonic shear shown in Fig. 5 has been increased by $120 \%$ to account for the discussed in Sec. IIIA.

The level of PCX signal recorded for the reversed shear plasma was lower than for the monotonic shear plasma. However the diagnostic was modified between the measurements reported here, so that we cannot verify quantitatively the ratio of predicted alpha densities. At both alpha energies and in both scenarios ORBIT calculations of profile shapes are in good agreement with the data.

\section{Discussion}

In both montonic and reversed shear scenarios we would expect the simulated profile at $0.5 \mathrm{MeV}$ to extend over a larger region in $\mathrm{r} / \mathrm{a}$ than at $1.3 \mathrm{MeV}$, as there is a larger stochastic confinement domain at lower energy. This is seen in the monotonic case. However, in reversed shear, Monte Carlo statistics near the plasma edge blur the distinction between the simulated profiles at 0.5 and $1.3 \mathrm{MeV}$. Several other features of the PCX analyzed data in reversed shear are distinctly different from the monotonic 
shear case. In the monotonic shear case, the PCX profiles have similar shapes at 0.5 $\mathrm{MeV}$ and 1.3 MeV. In reversed shear, the PCX data exhibit a broad distribution at 1.3 $\mathrm{MeV}$, and at $0.5 \mathrm{MeV}$ a profile more steeply peaked than in the monotonic case.

The model predicts the general characteristics of alpha loss in reversed shear clarifying these features. In reversed shear only a small fraction of trapped alphas would be observed at the birth energy $3.5 \mathrm{MeV}$. This is in contrast to the reversed shear scenarios of Ref. 3 for which no trapped alphas would be confined at $3.5 \mathrm{MeV}$. The effect of stochastic diffusion on the alpha particles in reversed shear at high q is to cause stochastic motion of the orbiting ion's banana tips within the confinement domain. In Fig. 7 and Fig. 8 are shown the motion of a $1.3 \mathrm{MeV}$ alpha particle initially at the midplane, at a radius given by edge normalized poloidal flux $=0.6$, with pitch $=0.3$ in the monotonic and reversed shear experimental scenarios. Note that the monotonic shear alpha orbits are largely unaffected by the presence of TF ripple, as the $1.3 \mathrm{MeV}$ alpha orbit is unchanged over 70 toroidal transits. In reversed shear, however, the banana tip undergoes rapid stochastic motion, driven by TF ripple at high q. This causes the average radial location of the alpha particle midplane crossing point to be broadened as those ions undergo stronger transport from TF ripple than in the monotonic shear case. The result as seen in the ORBIT simulations is a broadened profile at $1.3 \mathrm{MeV}$ in reversed shear and a peaked profile in montonic shear.

The pitch angle scattering rate is inversely dependent on the alpha energy, $v_{\mathrm{pa}} \sim$ $1 / \mathrm{E}^{3 / 2}$. At high energy, the trapped alpha distribution is not modified by pitch angle scattering and is only broadened by the effect of the stochastic motion. At lower energies the pitch angle scattering time drops from 20 seconds to $\sim 1$ second and becomes 
comparable to the time over which TF ripple acts on a lost alpha particle, so that the trapped alpha radial profile will reflect that of the total alpha particle distribution. The total alpha particle distribution will be largely characterised by the passing alpha particles, which are $\sim 2 / 3$ of the initial distribution and are not affected by TF ripple fields. The confinement domain grows in size as the ion energy decreases (Fig. 3). Once the alphas have slowed to energies at which, if trapped they are within the stochastic confinement domain, the trapped alpha density will accumulate and the stronger pitch angle scattering will cause it to reflect the radial profile of the full alpha distribution at that energy. The low threshold for alpha loss in reversed shear and the energy dependence of pitch angle scattering are responsible for the broader and lower alpha density in reversed compared to monotonic shear.

Gorelenkov [19] has carried out ORBIT simulations for a weak shear equilibrium with a narrowed pitch angle acceptance window, $-0.03<\lambda<-0.07$. He too finds a radial redistribution of alphas with high central q, possibly accounting for the observed low central alpha density at high energy in reversed shear [16].

If the central q were raised in the simulation to be much higher than the TRANSP predictions, there would be some difference in the stochastic ripple losses near $3.5 \mathrm{MeV}$ as the plasma would be above the critical threshold at that energy, as in the reversed shear scenario simulated in Ref. 3. The behavior of lower energy alphas would also be affected as reduction of the threshold would drive stochastic motion of the confined alphas. However, because $\mathrm{q}$ at the edge of the confinement domain would not be changed, lower energy alphas would not be lost. It is the effect of high average q, not high central q or 
reversed shear that causes large stochastic ripple transport of alphas in reversed shear plasma. As shown in Sec. IIID, high central q does lead to high first orbit losses.

\section{Comparison with alpha loss data}

Measurements with a scintillation detector at 90 degrees below the midplane during 1.6 MA reversed shear discharges showed the alpha loss per DT fusion neutron to be about three times larger than during standard 1.6 MA DT plasmas in the supershot regime [6]. This is consistent with the calculated increase in first orbit loss at the vessel bottom (6\% vs. $18 \%$ in Table II) due to the higher $\mathrm{q}(\mathrm{r})$ near the center.

A series of calculations was made to determine the relative importance of alpha profile width and $\mathrm{q}$ profile on the first orbit losses of these scenarios. ORBIT simulations of the reversed shear alpha profile, $\left(1-(\mathrm{r} / \mathrm{a})^{2}\right)^{5}$, with reversed and montonic shear q profiles led to $22 \%$ and $10 \%$ first orbit loss respectively. With the more peaked alpha profile of the monotonic shear case, $\left(1-(\mathrm{r} / \mathrm{a})^{2}\right)^{9}$, simulations with the reversed shear and monotonic shear $\mathrm{q}(\mathrm{r})$ led to $16 \%$ and $6 \%$ loss respectively. The differences in global loss fractions between these and the simulations shown in Table II are due to Monte Carlo noise (1-3\%). Here "first orbit loss" means losses within the time taken by a passing alpha at its birth energy to execute 25 toroidal transits ( 34 microseconds). Higher plasma $\mathrm{q}$ in the core of reversed shear discharges was primarily responsible for the high first orbit losses. A weak shear plasma having $\mathrm{q} \sim 2$ within $\mathrm{r} / \mathrm{a}=0.5$, would have larger poloidal flux at any radius than in the reversed shear case. Thus first orbit losses from a weak shear, high average q discharge would be lower than in reversed shear. 
Simulations of stochastic ripple diffusion predict that alpha loss due to banana diffusion will be peaked just below the plasma midplane [15]. Measurements of alpha loss with a scintillation detector at 20 degrees below the midplane were found to be larger at high $\mathrm{q}(0)$, but could not be interpreted in detail due to limiter shadowing effects [6].

\section{SECTION IV. CONCLUSION}

The dramatically improved thermal transport of reversed shear plasmas holds promise for future developments of magnetically controlled fusion. We find that neoclassical computational simulations of alpha particle density profiles are in good agreement with the PCX measurements of the alpha distribution function for both monotonic and reversed shear, in high fusion power plasmas on TFTR. The low threshold for alpha loss in reversed shear and the energy dependence of pitch angle scattering lead to a reduced alpha density and a broader alpha profile in reversed compared to monotonic shear. The guiding center code simulations presented here and in Ref. 2 for JT-60U confirm the value of predictive, first principles calculations of fast ion transport.

Fast ion losses predicted for toroidal confinement devices with high magnetic field ripple and high magnetic q will constrain the allowed magnetic field configurations of future reactors. The prediction of alpha losses can be even more critical for stellarator reactor designs. We have recently found that energetic particle ripple loss from new symmetric stellarator configurations is strongly dependent on the presence of pitch angle scattering, with an order of magnitude increase, when collisions are included in guiding center code simulations [20]. Although many difficult and challenging issues remain to 
be solved before fusion power is a practical reality, it is heartening that fast ion transport can be calculated on the basis of first principles. The exploitation of reversed shear in toroidal confinement reactors will require minimal magnetic field ripple and impurity levels to reduce collisional ripple loss and to optimize alpha particle confinement and heating.

\section{ACKNOWLEDGMENTS}

We are glad to acknowledge R. J. Goldston, R. J. Hawryluk and J. Hosea for their interest and encouragement. We also wish to thank H. Berk, U. of Texas at Austin, and N. Gorelenkov and R. Nazikian of PPPL for stimulating discussions on the redistribution of alphas by stochastic diffusion in reversed shear. Jill Testa, a summer research student at the Princeton Plasma Physics Laboratory during 1997 provided able assistance in the computer simulations. This research was supported by the U. S. Department of Energy Contract No. DE-AC02-76CH03073. 


\section{REFERENCES}

[1] R. J. Hawryluk, S. Batha, W. Blanchard, et al., Phys. Plas. 5, 1577 (1998).

[2] K. Tobita, H. Haruno, T. Nishitani, et al., Nuclear Fusion 37, 1583 (1997).

[3] M. H. Redi, R. B. White, S. H. Batha, F. M. Levinton, D. C. McCune, Phys, Plas. 4 4001 (1997).

[4] R. A. Fisher, J. M. McChesney, P. B. Parks, et al., Phys. Rev. Lett. 75846 (1995).

[5] S. M. Medley, D. K. Mansfield, A. L. Roquemore, et al., Rev. Sci. Instrum. 67, 3122 (1996).

[6] S. J. Zweben, D. S. Darrow, S. H. Batha, et al., Nuclear Fusion, 38739 (1998).

[7] R. J. Hawryluk, "Physics of Plasmas Close to Thermonuclear Conditions", ed. B. Coppi, et al, (CEC, Brussels, 1980), Vol. 1, p. 19.

[8] R. C. Grimm, J. M. Green, J. L. Johnson, Methods Comput. Phys. 16, 253 (1976).

[9] R. B. White and M. S. Chance, Phys. Fluids 27, 2455 (1984).

[10] R. B. White, R. J. Goldston, M. H. Redi, R. V. Budny, Phys. Plas. 3, 3043 (1996).

[11] R. B. White, Phys. Rev. E, 581774 (1998).

[12] R. J. Goldston, R. B. White, A. H. Boozer, Phys. Rev. Lett. 47647 (1981).

[13] M. H. Redi, R. V. Budny, D. S. Darrow, et al., Nuclear Fusion 35, 1509 (1995).

[14] R. V. Budny, M. G. Bell, A. C. Janos, et al., Nuclear Fusion 35, 1497 (1995).

[15] M. H. Redi, M. C. Zarnstorff, R. B. White, et al. Nuclear Fusion 35, 1191 (1995).

[16] M. H. Redi, S. H. Batha, R. V. Budny, et al., in Controlled Fusion and Plasma Physics (Proc. 24th Eur. Conf. Berchtesgaden, 1997), Vol. 21A, Part III, European Physical Society, Geneva (1997) 1069.

[17] S. M. Medley, R. V. Budny, H. H. Duong, et al., Nuclear Fusion, 38, 1283 (1998). 
[18] B. C. Stratton, R. V. Budny, D. S. Darrow, et al., IAEA-CN-69/OV4/2, Yokohama, Japan (1998).

[19] N. Gorelenkov, Princeton Plasma Physics Laboratory, private communication, 1998.

[20] M. H. Redi, H. E. Mynick, M. Suewattana, R. B. White, M. C. Zarnstorff, M. Yu. Isaev, M. I. Mikhailov, A. A. Subbotin, "Energetic particle transport in compact quasiaxisymmetric stellarators", submitted to Physics of Plasmas. 
TABLE I. Comparison of parameters for Ref. 3 scenarios and the PCX experiments

\begin{tabular}{|c|c|c|c|c|}
\hline \multicolumn{3}{|c|}{ Scenarios of Ref. $3^{\mathrm{a}}$} & \multicolumn{2}{|c|}{ PCX Experiments ${ }^{\mathrm{b}}$} \\
\hline \multicolumn{2}{|c|}{ Monotonic Shear } & Reversed Shear & Monotonic Shear & Reversed Shear \\
\hline $\mathrm{R}_{\mathrm{axis}}(\mathrm{m})$ & 2.8 & 3.0 & 2.7 & 2.7 \\
\hline $\mathrm{R}_{\text {geo }}(\mathrm{m})$ & 2.6 & 2.6 & 2.5 & 2.6 \\
\hline $\mathrm{a}(\mathrm{m})$ & 0.96 & 0.98 & 0.87 & 0.94 \\
\hline $\mathrm{BT}(\mathrm{T})$ & 4.8 & 4.6 & 4.9 & 4.6 \\
\hline$P_{\text {inj }}(M W)$ & 23 & 25 & 19 & 17 \\
\hline $\mathrm{I}_{\mathrm{p}}(\mathrm{MA})$ & 1.8 & 1.6 & 2.0 & 1.6 \\
\hline qo & 1.0 & 3.9 & 1.0 & 3.0 \\
\hline $\mathrm{q}_{\mathrm{a}}$ & 6.1 & 6.9 & 4.9 & 6.1 \\
\hline$\nu_{\mathrm{pa}}(/ \mathrm{sec})$ & 0.19 & 0.07 & 0.05 & 0.05 \\
\hline$v_{\varepsilon}(/ \mathrm{sec})$ & 3.3 & 13. & 3.4 & 4.4 \\
\hline \multicolumn{5}{|c|}{${ }^{a}$ Confinement domain and global loss calculations (Ref. 3) } \\
\hline \multicolumn{5}{|c|}{ and simulations of alpha radial profiles, this paper. } \\
\hline${ }^{\mathrm{b}}$ Confineme & th domain an & d global loss calcu & ions, this paper. & \\
\hline
\end{tabular}


TABLE II. Guiding center code simulations of alpha particle global loss for scenarios of Ref. 3 and PCX experiments

Scenarios of Ref. 3

PCX Experiments

Monotonic Shear Reversed Shear Monotonic Shear Reversed Shear
$(\%) \quad(\%)$
$(\%) \quad(\%)$

First Orbit

Delayed Particle Loss

Total Particle Loss

Total Energy Loss

$19 \quad 38$

$23 \quad 40$
$4 \quad 20$

$3 \quad 19$

$\begin{array}{ll}7 & 39\end{array}$

$6 \quad 36$ 


\section{FIGURE CAPTIONS}

Figure 1. Radial profiles of q used for simulations of experiments on TFTR near the end of neutral beam ion heating. The monotonic and reversed shear q profiles are shown for the Ref. 3 scenarios and the PCX experiments.

Figure 2. The $\mathrm{q}(\mathrm{r})$ radial profile measured with the MSE diagnostic after VMEC reconstruction for the reversed shear experiment in which PCX measurements were made. The measured $\mathrm{q}(\mathrm{r})$ profile is compared to the TRANSP prediction, at a time early in the beam heating phase. MSE data was not available for the corresponding monotonic experiment.

Figure 3. Stochastic confinement domains for alpha particles in the PCX experiments in monotonic and reversed shear scenarios at birth energy of $E_{o}=3.5 \mathrm{MeV}$, and energies of 1.3 MeV and $0.5 \mathrm{MeV}$ after one and two slowing down times. Toroidally symmetric poloidal cross sections of the plasma are shown. The dark region denotes the location of trapped ion bounce points at which the stochastic confinement criterion is met. Outside this region trapped alpha particles are rapidly lost due to prompt loss orbits and stochastic ripple diffusion.

Figure 4. Time evolution of global loss fractions for alpha particles from simulations of monotonic and reversed shear plasmas on TFTR over one alpha particle slowing down time for the PCX experiments.

Figure 5. Comparison of measured to calculated alpha particle densities for the monotonic shear scenario at the energies shown, within $\pm 70 \mathrm{keV}$ at the midplane and for a restricted window of pitch $\lambda=-0.05 \pm 0.001$. The PCX data points are benchmarked to the ORBIT simulations at the radius indicated. The absolute value of the alpha density 
is obtained from TRANSP simulation of the experiment, normalized as described in the text.

Figure 6. Comparison of measured to calculated alpha particle density for the reversed shear scenario at the energies shown, within $\pm 70 \mathrm{keV}$ at the midplane and for a restricted window of pitch $\lambda=-0.05 \pm 0.001$. The PCX data points are benchmarked to the ORBIT simulations at the radius indicated. The absolute value of the alpha density is obtained from TRANSP simulation of the experiment, normalized as described in the text.

Figure 7. Trapped particle orbits over 70 toroidal transits executed by a $1.3 \mathrm{MeV}$ alpha particle with $\lambda=-0.05$ in the monotonic shear PCX experiment.

Figure 8 . Trapped particle orbits over 70 toroidal transits executed by a $1.3 \mathrm{MeV}$ alpha particle $\lambda=-0.05$ in the reversed shear PCX experiment. 


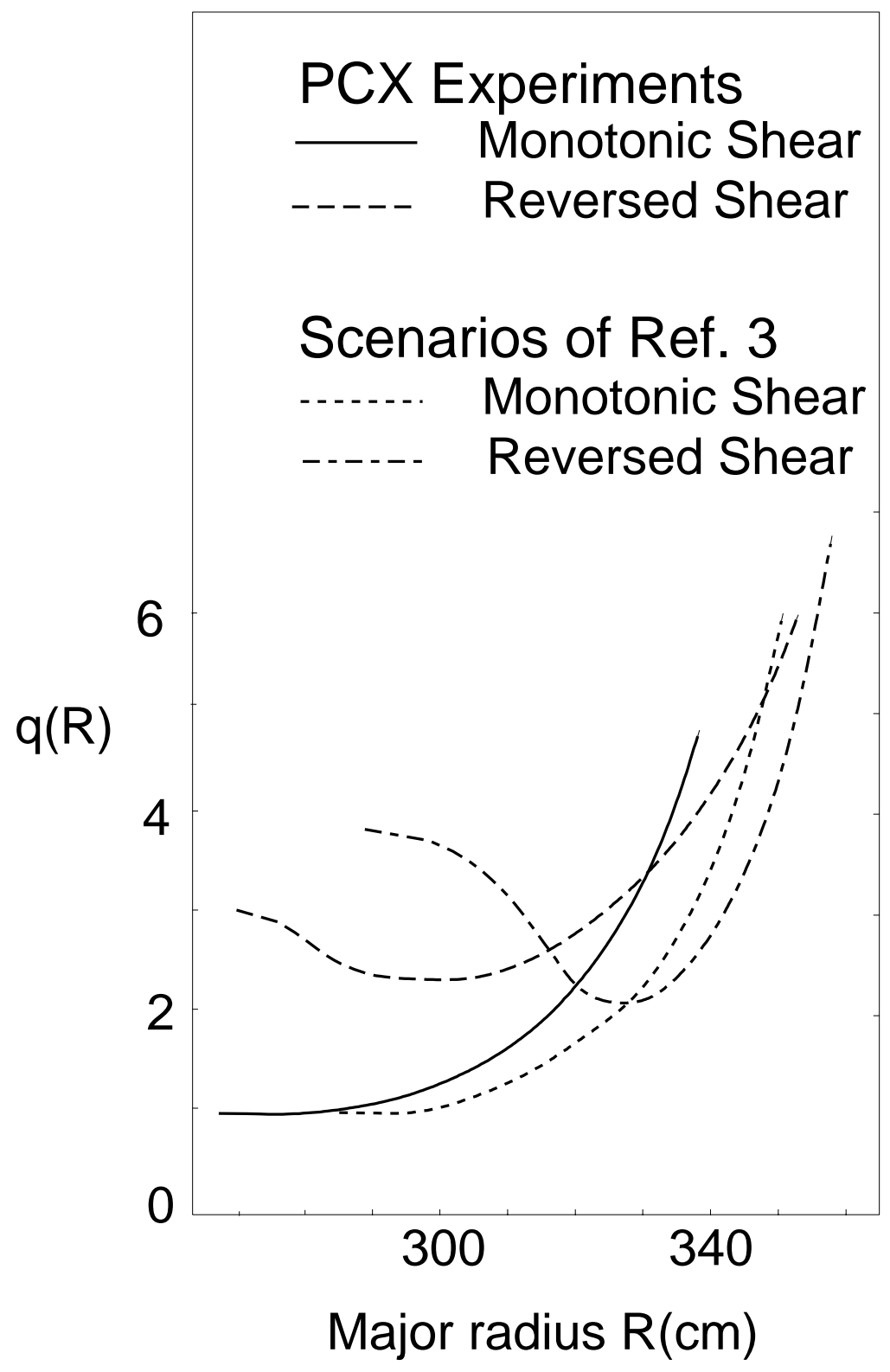

Fig. 1, Redi 


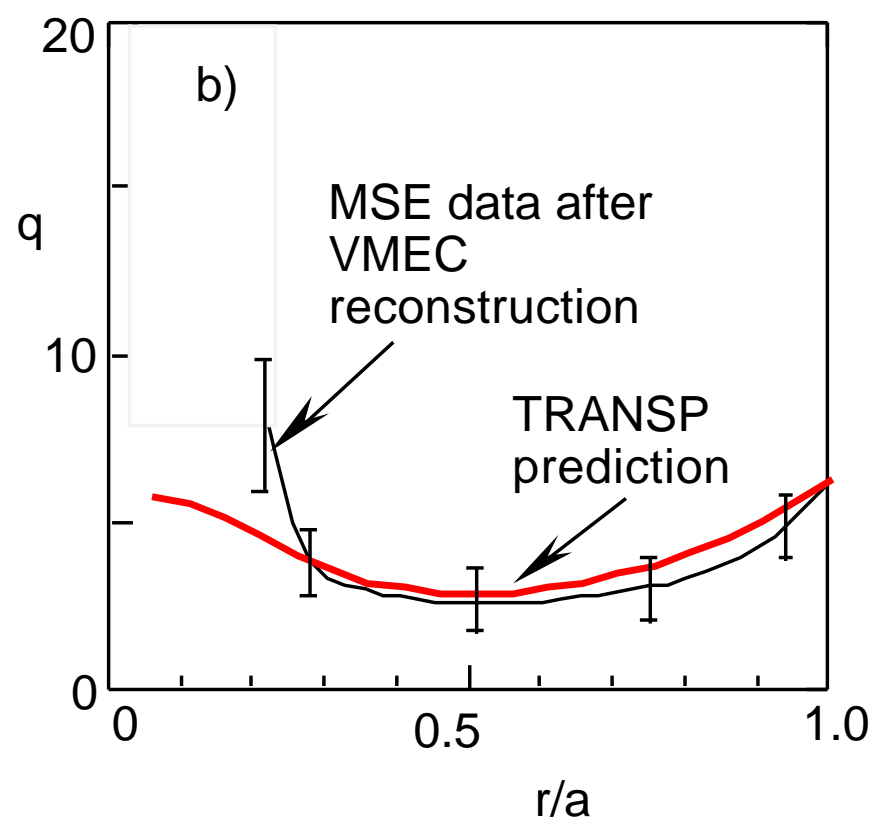

Figure 2, Redi 

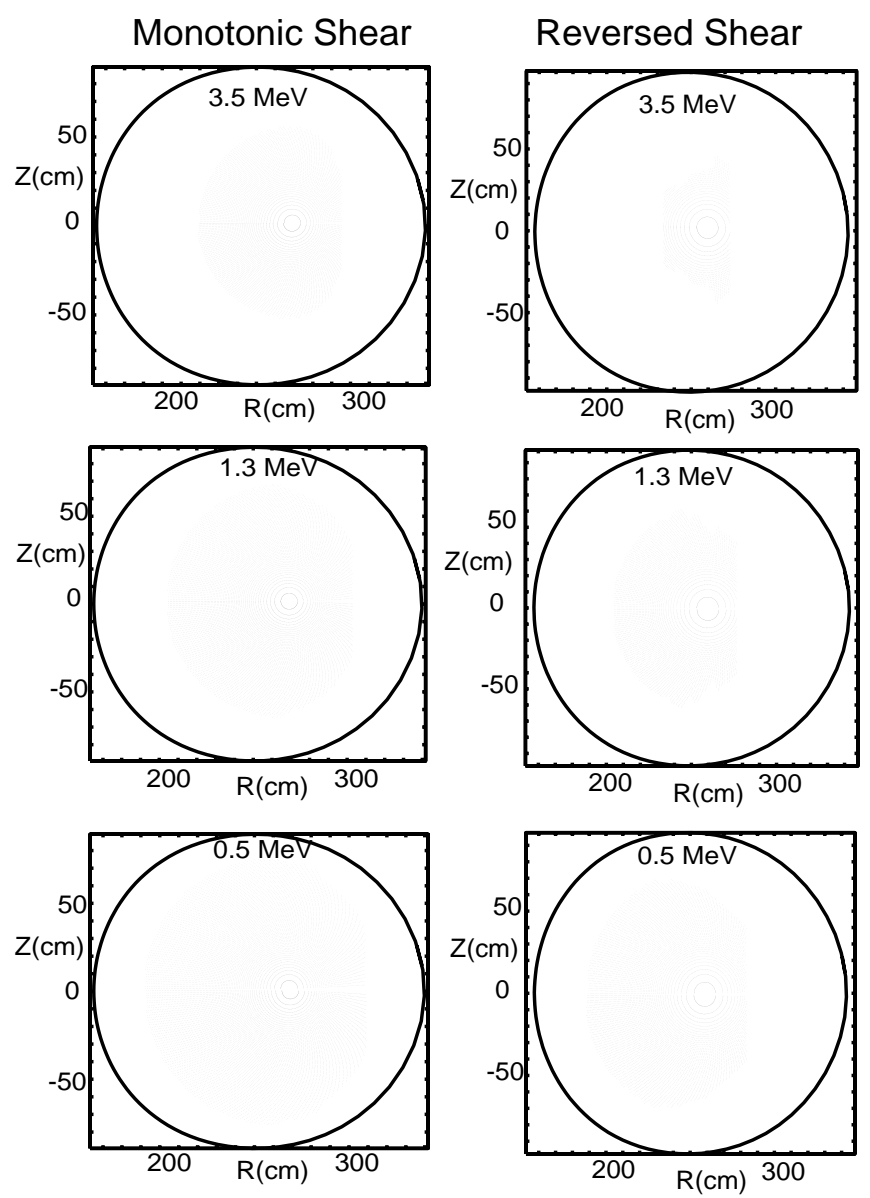

Fig. 3, Redi 


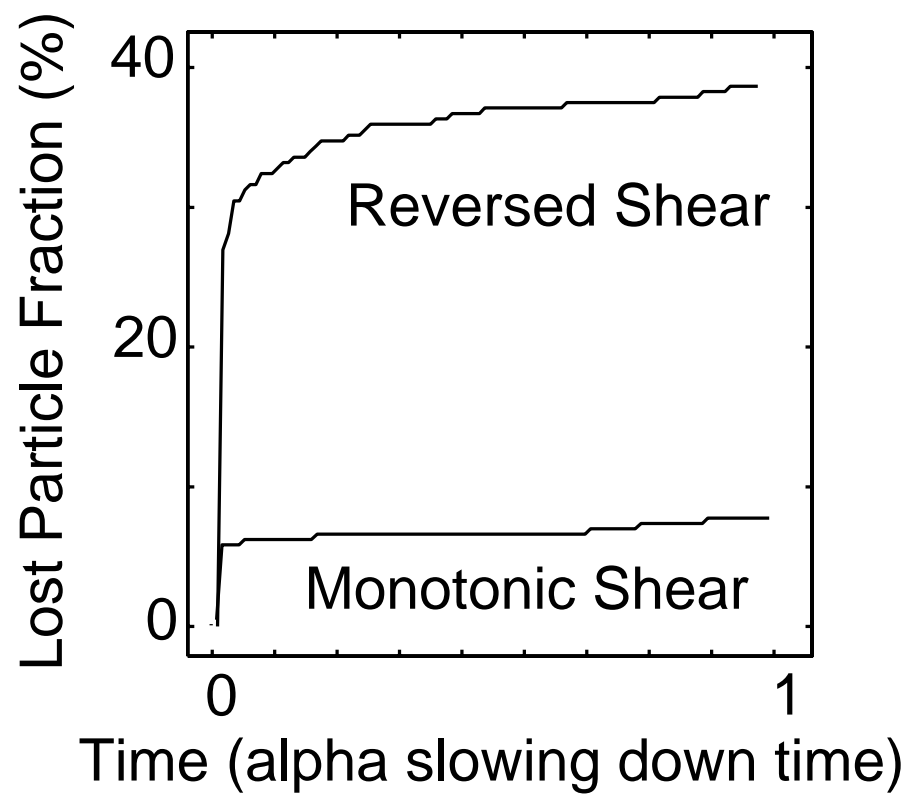

Fig. 4, Redi 


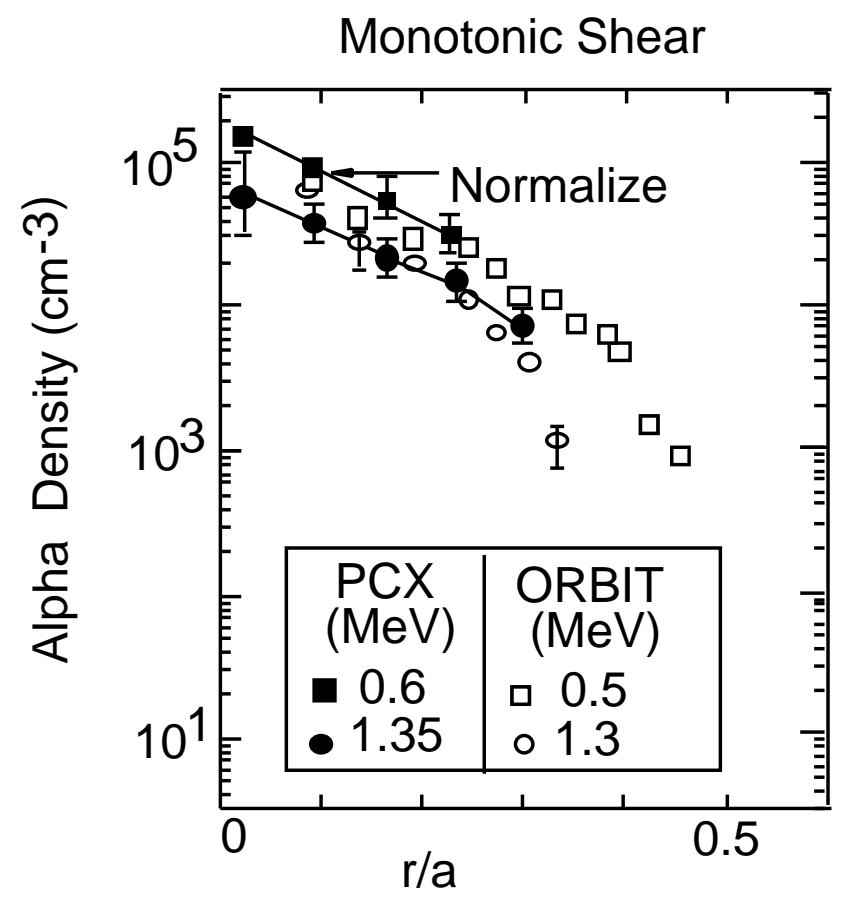

Figure 5, Redi 


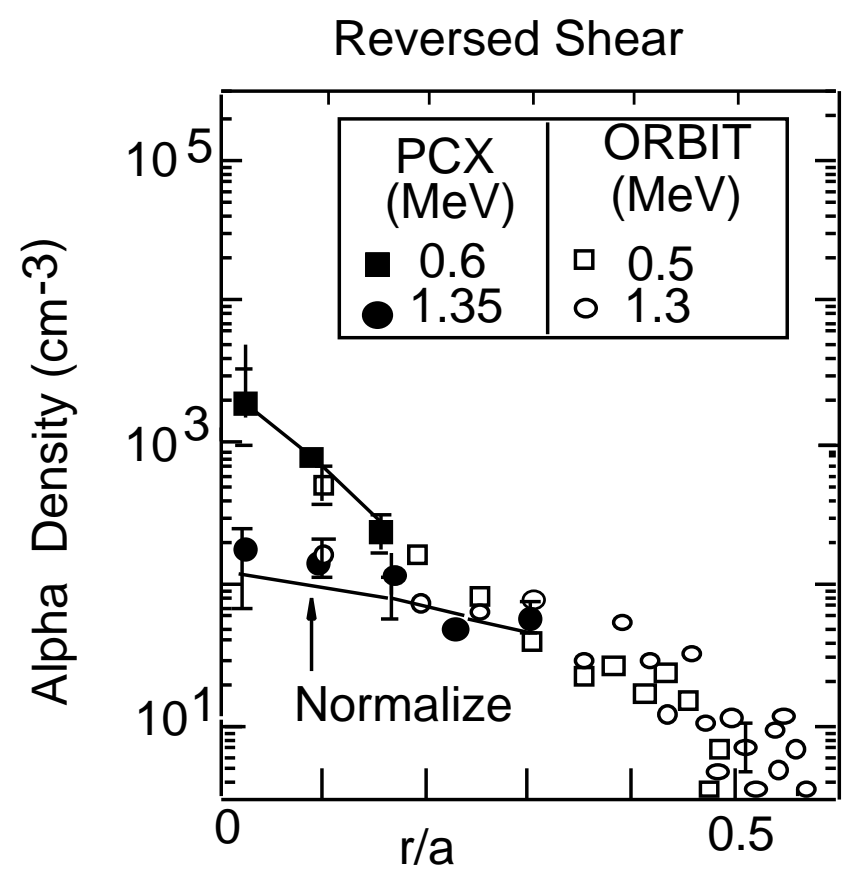

Figure 6,Redi 


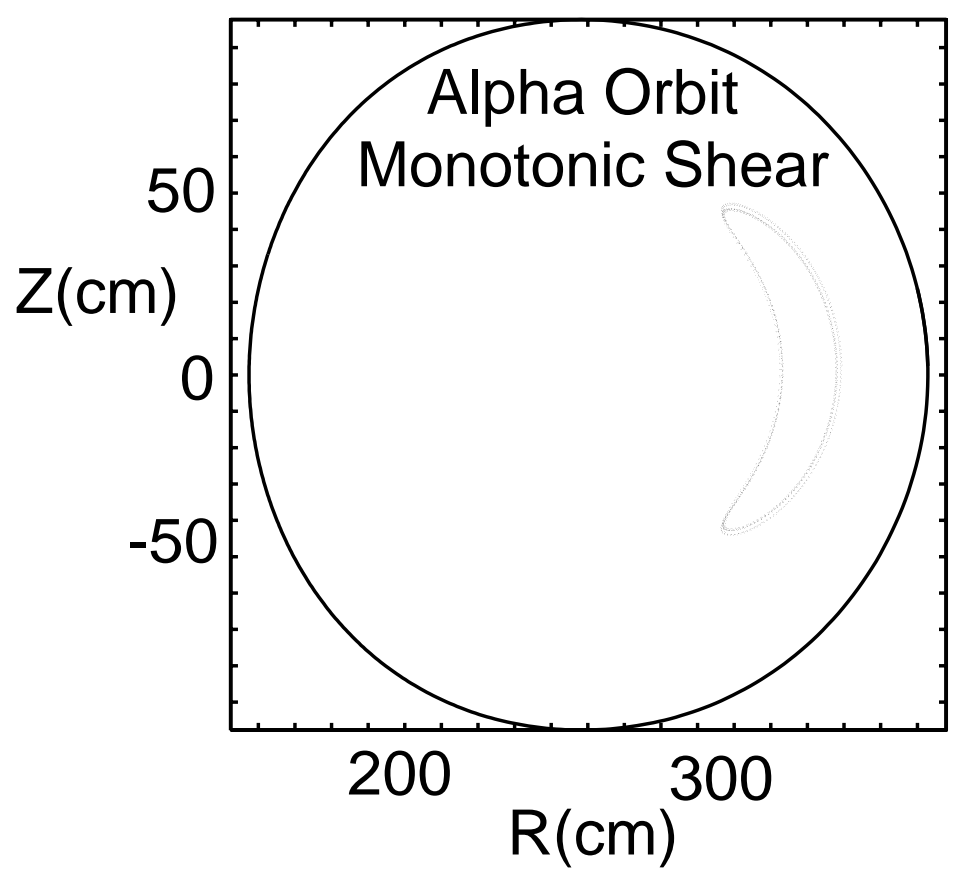

Redi, Figure 7 


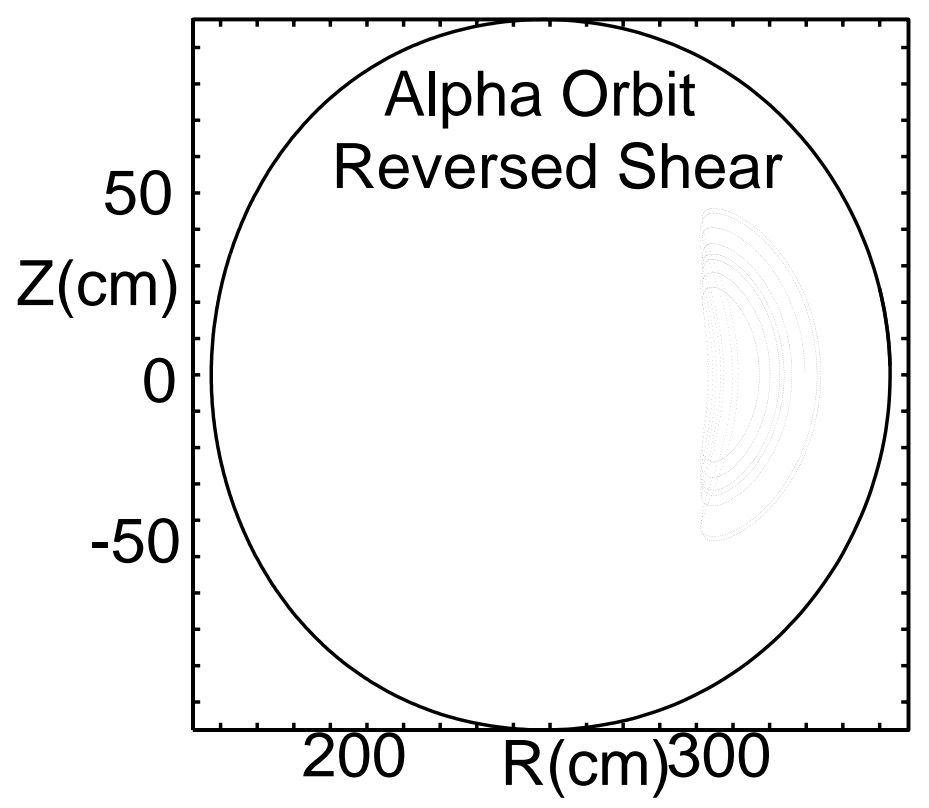

Redi, Figure 8 